\title{
Self-Tuning Control for Synchronous Machine Stabilization
}

\author{
Jozef Ritonja ${ }^{1}$ \\ ${ }^{1}$ Faculty of Electrical Engineering and Computer Science, University in Maribor, \\ Smetanova ulica 17, 2000 Maribor, Slovenia \\ jozef.ritonja@um.si
}

\begin{abstract}
A small-signal stability problem of a synchronous generator influencing a power grid was studied. A thorough theoretical analysis of a simplified linearized model of the synchronous generator connected to an infinite bus was carried out. The described analysis pointed out disadvantages of the conventional linear power system stabilizer. In contrast to the conventional, an original self-tuning power system stabilizer was developed. This stabilizer compacts a linear quadratic regulator and a recursive least square identification method. The study involving numerical simulations and laboratory experiments reveals encouraging results with displayed advantages and feasibility of this novel approach in considered applications.
\end{abstract}

Index Terms-Adaptive control, power system stability, synchronous machine.

\section{INTRODUCTION}

One of the most sophisticated control problems of a synchronous generator (SG) is the problem of small-signal stability. Oscillations of SG's electrical and mechanical variables reduce production capacity of SG, efficiency of a transmission line and lifespan of equipment. To enhance damping of SG and to improve the power system stability, SG must be equipped with a power system stabilizer (PSS) [1].

The oscillations of older SG with conspicuous damper windings have relatively small amplitudes. Therefore, these SG operate mostly without PSS. Due to cost-design construction optimization, modern SG have significantly less damper windings. This can in turn increase the oscillations and lead to the instability. In order to mitigate the stability problem and to ensure safe and economic operation, more and more of novel SG must be equipped with PSS [2].

The PSS utilize a static semiconductor's excitation system connected to rotor field winding. Conventional PSS design is based on a linear control theory. Conventional PSS is easy to realize. However, its application shows non-optimal damping throughout the operating range. Namely, by varying an operating point, SG's dynamic characteristics vary as well. This is the fact due to which PSS determined in the nominal operating point does not assure the optimal damping in the entire operating range. In the past decade, major modern control theories were tested for purposes of

Manuscript received November 16, 2014; accepted April 12, 2015. the PSS design. Of all the methods, the adaptive control has proved to be the most suitable for the design of PSS. The adaptive control has been used in order to assure the optimal damping through the entire SG operating range. The use of the adaptive control is possible because the loading variations and consequently the variations of the SG dynamic characteristics are, in most cases, essentially slower than the adaptation mechanism.

In this paper, the contribution to the stabilization of SG was carried out with the compact system of the self-tuning control using the linear quadratic regulator and the recursive least square identification. By studying its parts, this complex assembly looked promising and feasible for more effective stabilization of SG, and was the main motivation to take on. Subsequent research outcomes presented in this manuscript are encouraging and will also become a part of our lead in future development and real applications of the control within a commercial power plant. The main original contribution of the presented work is therefore description of the development and the advantages of new self-tuning PSS based on the linear quadratic regulator and the recursive least square identification method.

\section{MATHEMATICAL MODEL OF THE SYNCHRONOUS GENERATOR}

The SG connected to an infinite bus is a multivariable non-linear dynamic system described with a $7^{\text {th }}$ order nonlinear state-space model. For the control system design, a simplified linearized $3^{\text {rd }}$ order model (SLM) of SG is used [3]. SLM of SG has two inputs: a mechanical torque (from a turbine) and rotor field excitation voltage (from an excitation system); and three state-space variables: rotor speed, a rotor angle and voltage behind transient reactance. All variables in SLM denote deviations from an equilibrium state and are normalized on nominal base values.

During the normal operating conditions, SG is equipped with an automatic voltage regulator (AVR). The AVR controls SG's stator terminal voltage to be the same as reference terminal voltage. A disadvantage of the AVR control system is that an additional voltage control loop deteriorates stability of controlled SG [4]. The simplest model of AVR with the exciter is a first order lag.

A block diagram of SLM of SG with the voltage control system is shown in Fig. 1, where $T_{\mathrm{m}}$ is the mechanical torque $[\mathrm{pu}], T_{\mathrm{e}}$ is the electrical torque [pu], $\omega$ is the rotor speed 
[pu], $\delta$ is the rotor angle [rad], $E_{\mathrm{q}}^{\prime}$ is the voltage behind transient reactance [pu], $E_{\mathrm{fd}}$ is the field excitation voltage $[\mathrm{pu}], V_{\mathrm{t}}$ is the terminal voltage $[\mathrm{pu}], H$ is the inertia constant $[s], D$ is the damping coefficient of the damper windings $[\mathrm{pu} / \mathrm{pu}], \omega_{\mathrm{r}}$ is the nominal synchronous speed $\left[\mathrm{rad} s^{-1}\right], T_{\mathrm{d} 0}^{\prime}$ is the direct axis transient open circuit time constant $[s], K_{1}$ $\ldots K_{6}$ are the linearization parameters [pu/pu], $k_{\mathrm{AVR}}$ is the exciter and the voltage controller gain $[\mathrm{pu} / \mathrm{pu}], T_{\mathrm{AVR}}$ is the exciter time constant $[s]$ and $V_{\mathrm{t}, \mathrm{ref}}$ is the reference terminal voltage [pu]. Subscript denotes the deviation from an equilibrium state and $s$ is a Laplace complex variable.

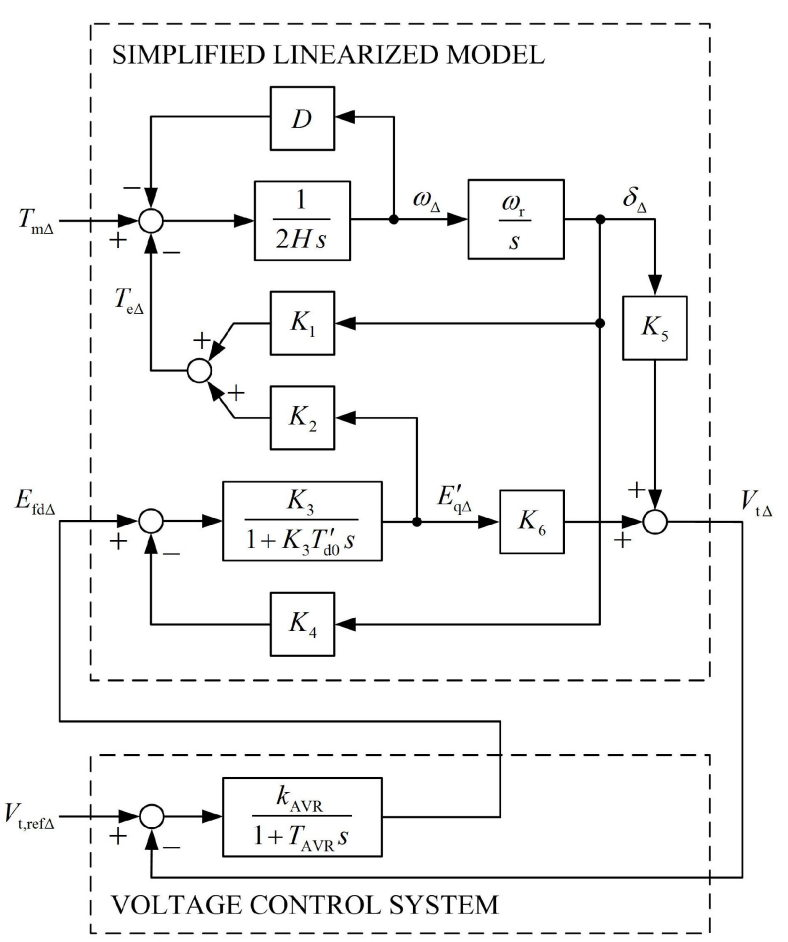

Fig. 1 Block diagram of simplified linearized model of synchronous generator connected to infinite bus, with voltage control system.

A state-space mathematical model of SG connected to the infinite bus together with the AVR control system is described with the following state-space equations:

$$
\begin{aligned}
{\left[\begin{array}{cc}
\dot{\omega} & (t) \\
\dot{\delta} & (t) \\
\dot{E}_{\mathrm{q}} & (t) \\
\dot{E}_{\mathrm{fd}} & (t)
\end{array}\right]=} & {\left[\begin{array}{cccc}
-\frac{D}{2 H} & -\frac{K_{1}}{2 H} & -\frac{K_{2}}{2 H} & 0 \\
\omega_{r} & 0 & 0 & 0 \\
0 & -\frac{K_{4}}{T_{\mathrm{d} 0}^{\prime}} & -\frac{1}{T_{\mathrm{d} 0}^{\prime} K_{3}} & \frac{1}{T_{\mathrm{d} 0}^{\prime}} \\
0 & -\frac{K_{5} k_{\mathrm{AVR}}}{T_{\mathrm{AVR}}} & -\frac{K_{6} k_{\mathrm{AVR}}}{T_{\mathrm{AVR}}} & -\frac{1}{T_{\mathrm{AVR}}}
\end{array}\right] \times } \\
& \times\left[\begin{array}{cc}
\omega & (t) \\
\delta & (t) \\
E_{\mathrm{q}} & (t) \\
E_{\mathrm{fd}} & (t)
\end{array}\right]+\left[\begin{array}{c}
0 \\
0 \\
0 \\
\frac{k_{\mathrm{AVR}}}{T_{\mathrm{AVR}}}
\end{array}\right] V_{\mathrm{t}, \mathrm{ref}}(t),
\end{aligned}
$$$$
\left[\begin{array}{cc}
\omega & (t) \\
\delta & (t) \\
V_{\mathrm{t}} & (t)
\end{array}\right]=\left[\begin{array}{cccc}
1 & 0 & 0 & 0 \\
0 & 1 & 0 & 0 \\
0 & K_{5} & K_{6} & 0
\end{array}\right]\left[\begin{array}{cc}
\omega & (t) \\
\delta & (t) \\
E_{\mathrm{q}} & (t) \\
E_{\mathrm{fd}} & (t)
\end{array}\right]+\left[\begin{array}{l}
0 \\
0 \\
0
\end{array}\right] V_{\mathrm{t}, \mathrm{ref}}
$$

In this paper, typical SG of a turbo type with $160 \mathrm{MVA}$ was utilized for: (-) the analysis by means of the numerical model, (-) for the control system design and synthesis, (-) as well as for the numerical simulations [1]. In references, such SG is commonly linked to benchmark cases. The data of this $\mathrm{SG}$ are presented in Table I, where $S_{\mathrm{n}}$ is the nominal power $[M V A], U_{\mathrm{n}}$ is the nominal voltage $[k V], \cos \varphi_{\mathrm{n}}$ is the nominal power factor, $x_{\mathrm{d}}$ and $x_{\mathrm{q}}$ are the unsaturated $d$ - and $q$-axis synchronous reactance $[\mathrm{pu}], x_{d}^{\prime}$ is the unsaturated $d$-axis transient reactance [pu], and $r_{\mathrm{e}}$ and $x_{\mathrm{e}}$ are the transmission line resistance and reactance $[\mathrm{pu}]$.

TABLE I. SYNCHRONOUS GENERATOR DATA FOR NUMERICAL ANALYSIS

\begin{tabular}{|c|c|c|c|}
\hline$S_{\mathrm{n}}=160 M V A$ & $U_{\mathrm{n}}=15 k V$ & $\cos \varphi_{\mathrm{n}}=0.85$ & $\omega_{\mathrm{r}}=377 s^{-1}$ \\
\hline$x_{\mathrm{d}}=1.7[\mathrm{pu}]$ & $x_{\mathrm{q}}=1.64[\mathrm{pu}]$ & $x_{d}^{\prime}=0.245[\mathrm{pu}]$ & $\begin{array}{c}T_{\mathrm{d} 0}^{\prime}=5.9 \\
s\end{array}$ \\
\hline$r_{\mathrm{e}}=0.02[\mathrm{pu}]$ & $x_{\mathrm{e}}=0.02[\mathrm{pu}]$ & $D=2[\mathrm{pu} / \mathrm{pu}]$ & $H=3.96 s$ \\
\hline & $T_{\mathrm{AVR}}=0.05[\mathrm{~s}]$ & $k_{\mathrm{AVR}}=59[\mathrm{pu} / \mathrm{pu}]$ & \\
\hline
\end{tabular}

\section{INFLUENCE OF THE SYNCHRONOUS GENERATOR OPERATING POINT ON THE LINEARIZED MODEL DYNAMICS}

By varying the operating point, the SLM's parameters $K_{1}$ through $K_{6}$ also vary. Numerical analysis of the benchmark SG shows that the values of the linearized parameters are changing significantly with a changing operating point. Figure 2 shows the influence of the operating point on the parameter $K_{5}$. The individual curve corresponds to the constant reactive power $Q$. On every individual curve the active power $P$ varies in the range from 0.0 to 1.2 [pu]. The individual curves correspond to the reactive powers from 0.0 to $1.2[\mathrm{pu}]$ in steps of $0.1[\mathrm{pu}]$.

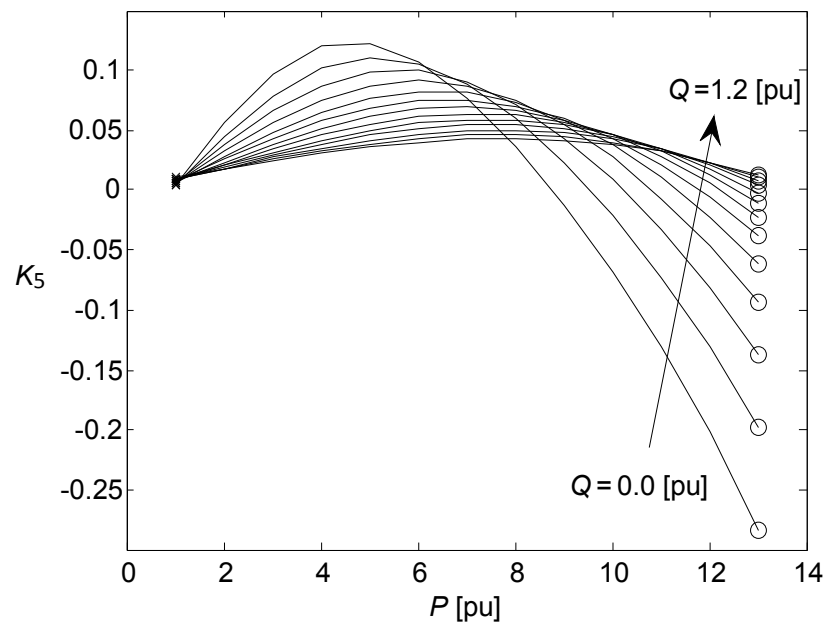

Fig. 2 Influence of operating point on parameter $K_{5}$.

The influence of variation of an operating point on other SLM's linearization parameters is similar to that in Fig. 2. The exception is parameter $K_{3}$ which is independent on the operating point variation.

For the SG stability analysis, parameters $K_{1}$ to $K_{6}$ are not sufficiently transparent [5]. The linearized model of SG with AVR, shown in (1) and (2) is a $4^{\text {th }}$ order linear model whose a characteristic polynomial has 4 eigenvalues. The influence of the operating point on the SG's dynamics can be clearly evaluated by an eigenvalue loci analysis. For the SG stability analysis, the dominant complex conjugate eigenvalues of the 
system matrix in (1) are especially interesting. They are directly related to the period and the damping ratio of the electromechanical oscillations. The results of the numerical eigenvalue analysis of benchmark SG for the same $P$ and $Q$ variations as in Fig. 2 are presented in Fig. 3.

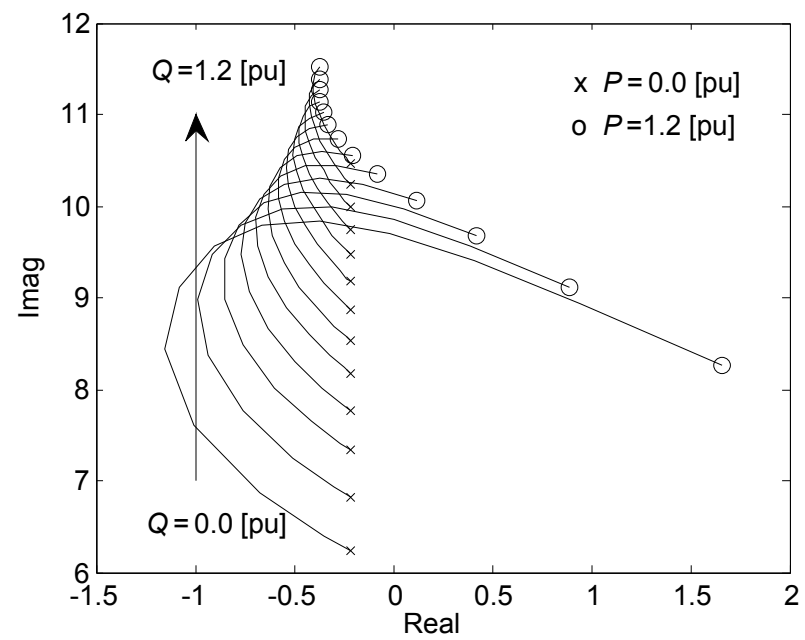

Fig. 3 Influence of operating point on dominant complex eigenvalue of simplified linearized model of SG with AVR.

From the analysis of the influence of the operating point variations on the SLM's eigenvalues, we can conclude that the variations in the SG dynamics are considerable and therefore implementation of adaptive PSS will be meaningful.

\section{Linear Quadratic POWER SySTEM STABILIZER}

Because of the non-linear dynamics of SG, conventional linear PSS does not assure optimal damping in the entire operating range. In this paper, the usage of adaptive PSS, based on a linear quadratic (LQ) state-space regulator is proposed. The input in the LQ regulator represents a state vector of the state-space mathematical model of SG with AVR, described in (1) and (2). The output of the LQ regulator will be connected to a summing junction of the voltage control system displayed in Fig. 1.

The LQ regulator is based on the state-space description of the linear dynamic system

$$
\dot{\mathbf{x}}(t)=\mathbf{A x}(t)+\mathbf{B u}(t),
$$

where $\mathbf{x}(t)$ is a $n$-vector of the state variables, $\mathbf{u}(t)$ is a $m$ vector of the input variables, $\mathbf{A}$ is the system matrix and $\mathbf{B}$ is the input matrix. All possible pairs $\mathbf{A}$ and $\mathbf{B}$ are controllable.

The objective of the control law is to drive the state vector $\mathbf{x}(t)$ from any non-zero initial values of states to the zero state vector in such a way that infinite-horizon quadratic cost function $J$ defined with (4) will be minimized [6]

$$
J=\int_{0}^{\infty}\left(\mathbf{x}^{\mathrm{T}}(t) \mathbf{Q} \mathbf{x}(t)+\mathbf{u}^{\mathrm{T}}(t) \mathbf{R} \mathbf{u}(t)\right) \mathrm{d} t
$$

In (4), $\mathbf{Q}$ is the symmetric positive semi-definite matrix and $\mathbf{R}$ is the symmetric positive definite matrix.

A feedback control law that minimizes cost function $J$ is defined as

$$
\mathbf{u}(t)=-\mathbf{K} \mathbf{x}(t),
$$

where $\mathbf{K}$ is the feedback gain given by

$$
\mathbf{K}=\mathbf{R}^{-1} \mathbf{B}^{\mathrm{T}} \mathbf{P}
$$

and $\mathbf{P}$ is found by solving the algebraic Riccati equation

$$
\mathbf{A}^{\mathrm{T}} \mathbf{P}+\mathbf{P A}-\mathbf{P B R} \mathbf{R}^{-1} \mathbf{B}^{\mathrm{T}} \mathbf{P}+\mathbf{Q}=\mathbf{0} .
$$

\section{SELF-TUNING POWER System StABILIZER}

Self-tuning PSS performs tuning of the PSS parameters at the start of the operation and the adaptation of the PSS parameters to the changeable dynamics during the operation [7]. In such a way, self-tuning PSS assures optimal damping of the SG's electromechanical oscillations in the entire operating range. Self-tuning PSS consists of four modules, such as:

- decision mechanism module,

- plant identification module,

- parameter tuning module, and

- controller implementation module.

A block diagram of self-tuning PSS is shown in Fig. 4. The modules will be only briefly described.

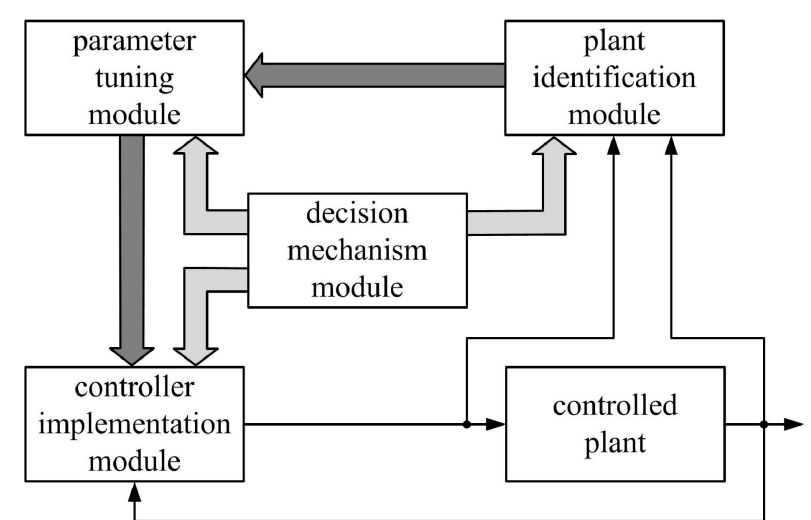

Fig. 4 Block diagram of self-tuning PSS control system.

\section{A. Decision Mechanism Module}

A Decision mechanism module coordinates operation of the other three modules. During the operation, this module performs continuous observation of SG's oscillations. Based on this information, the decision mechanism module controls the following main tasks:

- controlled plant identification,

- controller parameters calculation and

- control law implementation.

\section{B. Plant Identification Module}

The parameters of the state-space mathematical model of SG must be known to calculate the parameters of the LQ regulator. A plant identification module identifies parameters of the mathematical model of the controlled plant [8]. To implement the identification, the decision mechanism module coordinates the activities of the controller implementation module and the plant identification module. The controller implementation module generates a supplementary pseudo random binary signal for controlled 
plant input perturbation. On a basis of these input changes, the plant identification module calculates a discrete transfer function of the controlled plant. A well-known recursive least square method was used for parametric identification [9].

\section{Parameter Tuning Module}

A parameter tuning module calculates feedback gain $\mathbf{K}$ of the LQ regulator from the identified discrete transfer function. The procedure to calculate feedback gain $\mathbf{K}$ consists of two parts.

First, from the identified discrete transfer function, the continuous state-space model of the controlled plant as described in [10] is calculated.

Then, from the state-space model of the controlled plant (1), the parameters of the LQ regulator are calculated.

\section{Controller Implementation Module}

The controller implementation module executes the control law described by (5).

\section{Simulation RESUlTS}

To evaluate the applicability of developed self-tuning PSS, proposed PSS was numerically tested. The control system design was carried out for the linearized model of SG with AVR. For the evaluation of the control system performances under small-signal and large-signal disturbances, the $7^{\text {th }}$ order non-linear model of SG was used [1]. Deterministic and stochastic disturbances were systematically evaluated.

Simulations were conducted in the entire operating range with more than 100 operating points. Due to the volume of the paper, only results of the two typical operating points are presented: the nominal operating point and very weakly damped real operating point. The analysed operating points and the corresponding eigenvalues of SLM of SG with AVR are presented in Table II.

TABLE II. PRESENTED OPERATING POINTS WITH CORRESPONDING EIGENVALUES

\begin{tabular}{|c|c|}
\hline \multicolumn{2}{|c|}{ Operating point: $P=1.0[\mathrm{pu}], \cos \varphi=0.85$} \\
\hline$\lambda_{1,2}=-0.492 \pm 10.665 \mathrm{i}$ & $\lambda_{3,4}=-9.995 \pm 2.535 \mathrm{i}$ \\
\hline \multicolumn{2}{|c|}{ Operating point: $P=0.5[\mathrm{pu}], \cos \varphi=0.5$} \\
\hline$\lambda_{1,2}=-0.385 \pm 10.119 \mathrm{i}$ & $\lambda_{3,4}=-4.880 \pm 10.101 \mathrm{i}$ \\
\hline
\end{tabular}

Input changes are the same in all the cases and are shown in Fig. 5. The variation of the SG's electrical loads has random step changes. In the voltage measurement system and in the field excitation system, a band limited white noise signal with height of power spectral density PSD $=0.0015$ was introduced.



(a)

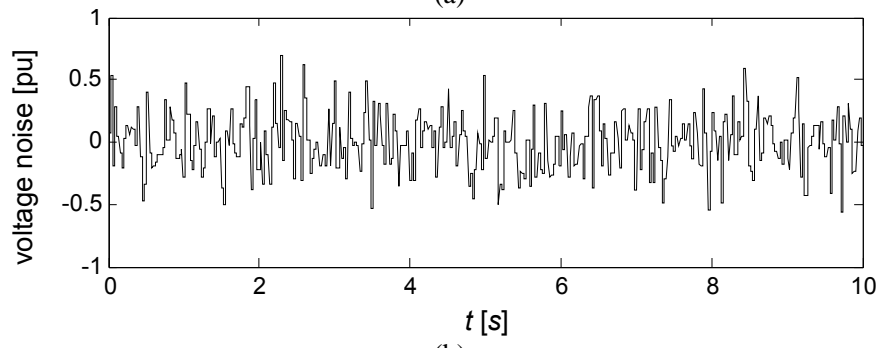

(b)

Fig. 5 Input perturbations: electrical load [pu] (a) and voltage disturbances with noise [pu] (b).

Figure 6 shows the response of the SG rotor speed on the described perturbations in the nominal operating point without use of PSS. As can be observed in Fig 6, the response of SG without PSS is poorly damped, hence it is inevitable to use PSS.

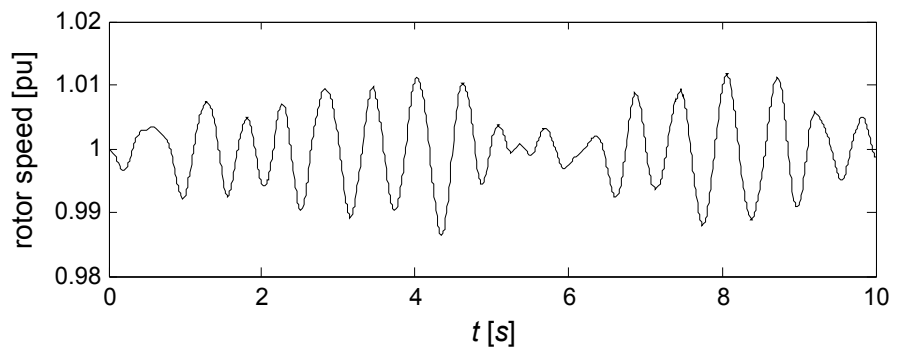

Fig. 6 Rotor speed in nominal operating point, without PSS. 


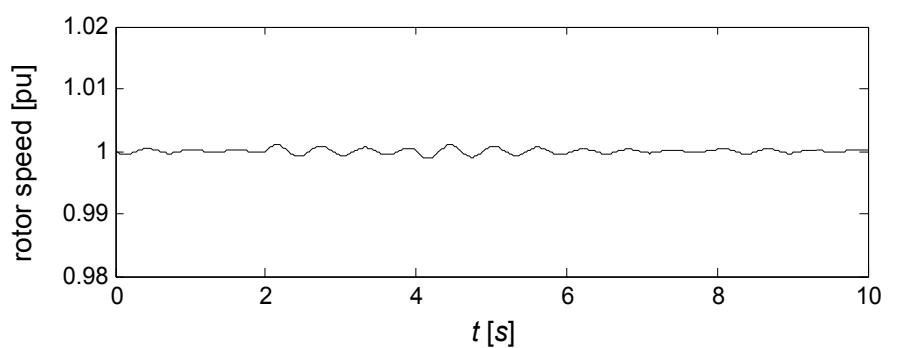

(a)

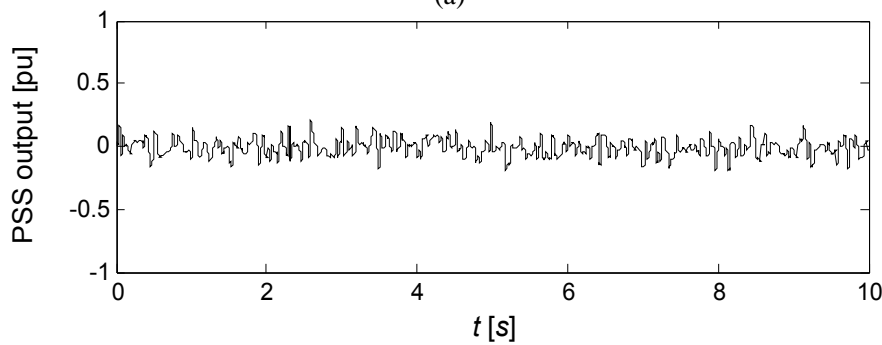

(b)

Fig. 7 Rotor speed and stabilizer output in nominal operating point $P=1.0[\mathrm{pu}]$ (a) and $\cos \varphi=0.85$ with self-tuning PSS (b).

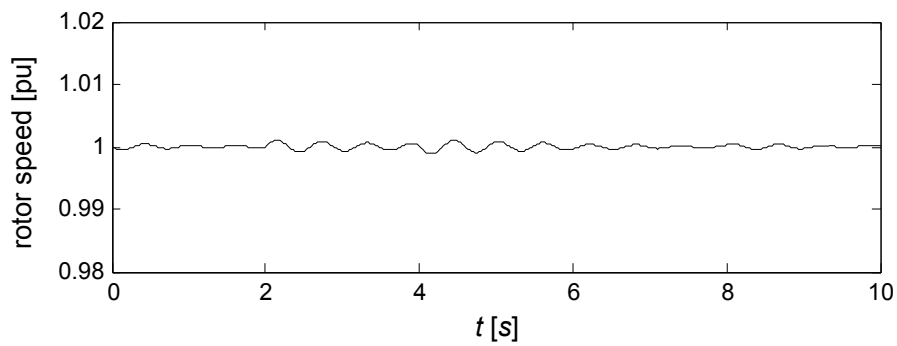

(a)

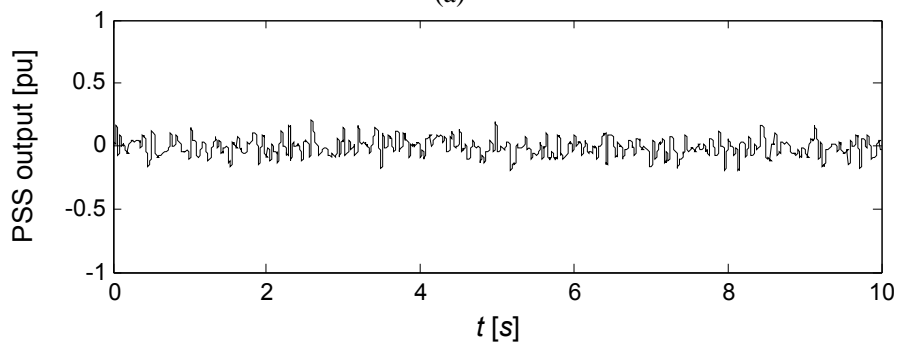

(b)

Fig. 8 Rotor speed and stabilizer output in weakly damped operating point $P=0.5[\mathrm{pu}$ (a) and $\cos \varphi=0.5$ with self-tuning PSS (b).

Figure 7 shows behaviour of SG in the nominal operating point where self-tuning PSS was used. The figure presents the response of the rotor speed and the PSS output signal on the same input disturbances as in Fig. 6. Controlled plant identification and controller parameter tuning were carried out previously. As can be observed in Fig. 7, the damping of SG with self-tuning PSS was significantly improved - the amplitudes of the oscillations with self-tuning PSS are more than 10 times smaller than in the case without PSS.

Figure 8 shows behaviour of SG in the weakly damped operating point. Such the operating point represents real weakly damped operation of SG in the power plant. The controller with the parameters obtained for the nominal operating point does not assure optimal damping in a new operating point. In this case, the decision mechanism module started the plant identification and tuning of the controller parameters. The LQ regulator in the controller implementation module assures optimal damping in a new operating point, which can be observed in Fig. 8.

\section{EXPERIMENTAL RESULTS}

The proposed self-tuning controller has been tested on 15 kVA SG connected to the electrical network. Tested SG has the following data:

TABLE III. DATA OF LABORATORY TESTED SYNCHRONOUS

\begin{tabular}{|c|c|c|}
\hline \multicolumn{3}{l}{ GENERATOR. } \\
\hline$S_{\mathrm{n}}=15 \mathrm{kVA}$ & $U_{\mathrm{n}}=400 \mathrm{~V}$ & $I_{\mathrm{n}}=21.7 \mathrm{~A}$ \\
\hline $\cos \varphi_{\mathrm{n}}=0.80$ & $f=50 \mathrm{~Hz}$ & $n_{\mathrm{n}}=1500 \mathrm{r} / \mathrm{min}$ \\
\hline
\end{tabular}

Instead of a turbine, a $30 \mathrm{~kW}$ AC motor with a frequency converter was used for generation of a mechanical torque. A static excitation system was used to generate field excitation rotor voltage. The control system was realized with dSpace. Figure 9 shows the experimental connection of the tested synchronous generator with the mechanical torque generating AC motor. 


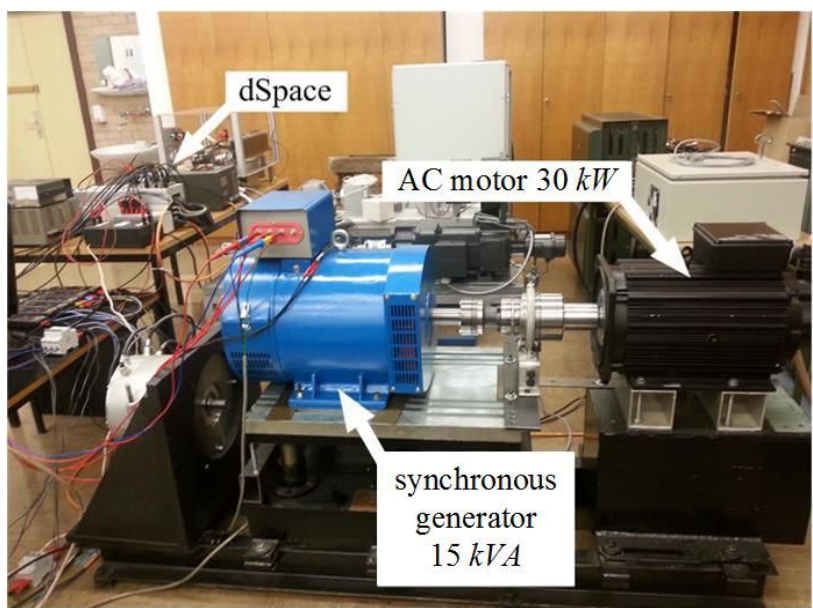

Fig. 9 Tested synchronous generator with mechanical torque generated by AC motor.

For the controlled plant identification, the SG's inputs must be changed to produce perturbations in SG's behaviour. For this reason, the step changes in the $\mathrm{AC}$ motor's mechanical torque and in the static excitation system's voltage were generated. On a basis of these perturbations, the discrete transfer functions of the controlled plant were calculated. The identification time of the recursive least square method is dependent on the magnitude and the dynamics of the input signals, on the forgetting factor and on the initial matrix of the identification method [9]. The SG was identified in the entire operating range. In all the cases, the identification time was less than 10 seconds. From identified transfer functions, the state-space mathematical models for different operating points were calculated. These models were used for the calculations of the LQ-regulator parameters.

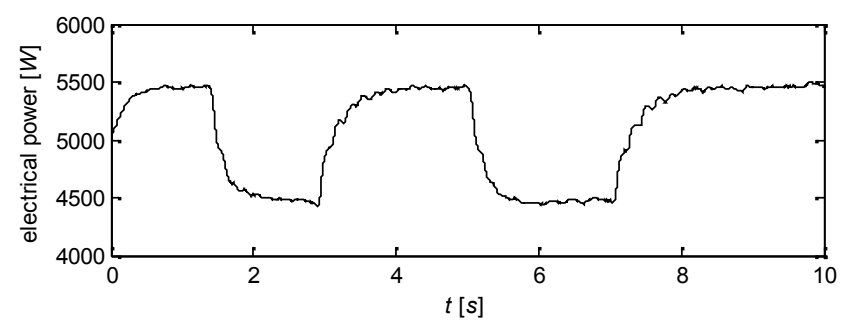

Fig. 10 Response of tested synchronous generator electrical power following step changes of AC motor's mechanical torque.

The calculated controller parameters improve damping of $\mathrm{SG}$ in the entire operating range. Figure 10 presents response of SG following the step changes of the AC motor's mechanical torque in the vicinity of the operating point of $5 \mathrm{~kW}$ electrical power. Similar well damped responses were obtained in the entire operating range. In all the cases laboratory tests confirm the findings of the numerical calculations. The recursive least square identification method has proved to be very convenient for the identification of the synchronous generator. The calculation of the LQ-regulator parameters from the state-space mathematical model of SG is computationally undemanding. The LQ-regulator has well shown elimination of the low frequency disturbances and the high frequency noise signal.

\section{CONCLUSIONS}

Due to changes in construction of novel SG, development of more effective PSS is inevitable. The paper presents the development and the application of self-tuning PSS for the improvement of the SG's small-signal stability. Proposed self-tuning PSS is based on the coordination of the recursive least square identification method and the LQ-regulator. The combination of the selected identification and control methods proved to be applicable for the PSS design. Presented self-tuning PSS makes the initial tuning of the controller parameters easier and assures the damping improvement in the complete operating range. The results of theoretical simulations and laboratory tests show essential improvement of the SG damping. Due to the encouraging outcomes, it is incumbent on the study it will also head towards real applications (a power plant anticipated).

The discussed problem is up to date. The development of new PSS is intense. We estimate that in the near future the advanced PSS concepts will replace conventional PSS in commercial excitation systems.

\section{REFERENCES}

[1] P. M. Anderson, A. A. Fouad, Power system control and stability. Ames, Iowa: The Iowa State University Press. 1977.

[2] P. Kundur, Power system stability and control. New York: McGrawHill, Inc., 1994.

[3] J. Machowsky, J. W. Bialek, J. R. Bumby, Power system dynamics: stability and control. Chichester: John Wiley \& Sons, 2008.

[4] A. Jonaitis, "Impact of increased frequency excitation system on stability of synchronous generator", Elektronika ir Elektrotechnika, vol. 19, no. 4, pp. 29-32, 2013. [Online]. Available: http://dx.doi.org/ 10.5755/j01.eee.19.4.2578

[5] J. Ritonja, "Adaptive stabilization for generator excitation system", Compel, vol. 30, no. 3, pp. 1092-1108, 2010.

[6] A. Sinho, Linear systems: Optimal and robust control. New York: Taylor \&Francis Group, 2007.

[7] R. R. Bitmead, M. Gevers, V. Wertz, Adaptive optimal control. New York: Prentice Hall, 1990.

[8] A. Jonaitis, J. Daunoras, "Identification of dynamic model of synchronous generator high frequency excitation", Elektronika ir Elektrotechnika, no. 1, pp. 25-28, 2011.

[9] R. Isermann, M. Muenchhof, Identification of dynamic systems. Berlin: Springer Verlag, 2011. [Online]. Available: http://dx.doi.org/ 10.1007/978-3-540-78879-9

[10] M. Karrari, O. P. Malik, "Identification of physical parameters of a synchronous generator from online measurement", IEEE Trans. on Energy Conversion, vol. 19, no. 2, pp. 407-415, 2004. [Online]. Available: http://dx.doi.org/10.1109/TEC.2003.822296 